\title{
PRESSURE DROP OF HFE7000 AND HFE7100 IN FLOW CONDENSATION IN MINICHANNELS WITH ACCOUNT OF NON-ADIABATIC EFFECTS
}

\author{
Dariusz Mikielewicz $^{1, a}$, Rafał Andrzejczyk ${ }^{1}$ and Jarosław Mikielewicz ${ }^{2}$ \\ ${ }^{1}$ Gdansk University of Technology, Faculty of Mechanical Engineering, Gdansk, Poland \\ ${ }^{2}$ The Szewalski Institute of Fluid-Flow Machinery PAS, ul. Fiszera 14, 80-231 Gdansk, Poland \\ E-mail : Dariusz.Mikielewicz@pg.gda.pl, rafal.andrzejczyk@pg.gda.pl, jarekm@imp.gda.pl
}

\begin{abstract}
Flow boiling and flow condensation are often regarded as two opposite or symmetrical phenomena involving the change of phase. There is a temptation to describe both these phenomena with one only correlation. From amongst the structures present in flow boiling and flow condensation at least the annular flow structure seems to be mostly appropriate to the common modeling. However, the shear stress acting between vapor phase and liquid phase is not the same in the respective cases, i.e. flow boiling and flow condensation. Most of modeling of heat transfer in case of condensation inside channels relates the heat transfer coefficient to the friction coefficient. All existing approaches are either the empirical fits to experimental data or form an attempt to combine two major influences to heat transfer, namely the convective flow boiling without bubble generation and nucleate boiling. In the paper the authors present investigations of flow condensation with the use of the HFE7100 and HFE 7000 as a working fluids and their own condensation model inside tubes with account of non-adiabatic effects. The model will be confronted with own data for a new fluid HFE7000 and HFE 7100.
\end{abstract}

\section{Introduction}

Flow boiling and flow condensation are often regarded as two opposite or symmetrical phenomena involving the change of phase. There is a temptation to describe both these phenomena with one only correlation, however such method has yet to be suggested. The objective of the present paper is to present modeling of flow boiling and condensation. The shear stress between vapor phase and liquid phase is generally a function of non-adiabatic effects. That is a major reason why that up to date approaches, considering the issue of flow boiling and flow condensation as symmetric, are failing in that respect. two opposite or symmetrical phenomena involving the change of phase. The way forward is to incorporate a mechanism into the convective boiling term responsible for modification of shear stresses at the vapor-liquid interface. First, the capability of the flow boiling model, developed earlier by the authors, extended to model also the case of condensation inside tubes, is discussed. The first modification to the calculation model

\footnotetext{
${ }^{\mathrm{a}}$ Corresponding author: Dariusz.Mikielewicz@pg.gda.pl
}

regards the change of the liquid film thickness in the annular flow structure. That is modeled using the so called "blowing parameter", which modifies the shear stress in annular flow. Another modification accounts for the influence of heat flux in the bubbly flow. As our objective is to devise a general model applicable both to flow boiling and flow condensation we will attempt to improve the model presented earlier in [2] to include the outlined above effects. Incorporation of the so called "blowing parameter", B, contributes to the liquid film thickening in case of flow condensation and thinning in case of flow boiling. The predictions obtained using authors own model will be confronted with obtained inhouse experimental data collected recently for HFE 7100 and HFE 7000.Nowadays an ecological justification causes a rapid development of refrigerating fluids so there is continuous need for verifying the research carried out up to now in the new conditions. Amongst new fluids can be found the Novec fluid HFE 7100 and HFE 7000. Its perspective area of application are low and medium temperature organic Rankine cycles. This fluids are the 
first of a new generation of low-GWP dielectric fluids with many of the properties of perfluorinated liquids. Other areas of application include its use in many traditional applications such as electric cooling, food refrigeration, industrial cooling, thermal management of semiconductors, etc.

\section{Two-phase pressure drop model based on dissipation}

Flow resistance due to friction is greater than in case of single phase flow with the same flow rate. The two-phase flow multiplier is defined as a ratio of pressure drop in two-phase flow, $(\mathrm{dp} / \mathrm{dz})_{\mathrm{TP}}$, to the total pressure drop in the flow with either liquid of vapor, $(\mathrm{dp} / \mathrm{dz})$, present:

$$
\Phi^{2}=\left(\frac{d p}{d z}\right)_{T P}\left(\frac{d p}{d z}\right)^{-1}
$$

\subsection{Dissipation based model for pressure drop calculations}

The fundamental hypothesis in the model under scrutiny here is the fact that the dissipation in two-phase flow can be modeled as a sum of two contributions, namely the energy dissipation due to shearing flow without the bubbles, $\mathrm{E}_{\mathrm{TP}}$, and dissipation resulting from the bubble generation, $\mathrm{E}_{\mathrm{PB}}$, Mikielewicz [5]:

$$
E_{T P B}=E_{T P}+E_{P B}
$$

Dissipation energy is expressed as power lost in the control volume. The term power refers to compensation of two-phase flow friction losses and is expressed through a product of shear stress and flow velocity. Analogically can be expressed the energy dissipation due to bubble generation in the two-phase flow. A geometrical relation between the friction factor in twophase flow is obtained which forms a geometrical sum of two contributions, namely the friction factor due to the shearing flow without bubbles and the friction factor due to generation of bubbles, in the form:

$$
\xi_{T P B}^{2}=\xi_{T P}^{2}+\xi_{P B}^{2}
$$

In the considered case $\xi_{\mathrm{PB}}$ is prone to be dependent on applied wall heat flux. As can be seem from (3) the friction factors in two phase flow are summed up in a geometrical manner. The first term on the right hand side of (3) can be determined from the definition of the twophase flow multiplier (1). Pressure drop in the two-phase flow without bubble generation can also be considered as a pressure drop in the equivalent flow of a fluid flowing with velocity $\mathrm{w}_{\text {TP. }}$. The pressure drop of the liquid flowing alone can be determined from a corresponding single phase flow relation. In case of turbulent flow we will use the Blasius equation for determination of the friction factor, whereas in case of laminar flow the friction factor can be evaluated from the corresponding expression valid in the laminar regime. A critical difference of the method (1) in comparison to other authors models is incorporation of the two-phase flow multiplier into modeling. There are specific effects related to the shear stress modifications, named here the non-adiabatic effects, which will be described below. One of the effects is pertinent to annular flows, whereas the other one to the bubbly flow.

\subsection{Non-adiabatic effects in annular flow}

The shear stress between vapor phase and liquid phase is generally a function of non-adiabatic effects. That is a major reason why up to date approaches, considering the issue of flow boiling and flow condensation as symmetric phenomena, are failing in that respect. The way forward is to incorporate a mechanism into the convective term responsible for modification of shear stresses at the vapor-liquid interface. We will attempt now to improve the shear stress between liquid and vapor phase in annular flow by incorporation of the so called "blowing parameter", B, which contributes to the liquid film thickening in case of flow condensation and thinning in case of flow boiling, Mikielewicz (1978). The formula for modification of shear stresses in the boundary layer reads:

$$
\tau^{+}=1+\frac{B}{\tau_{0}^{+}} u^{+}
$$

In (4) $\tau^{+}=\tau / \tau_{\mathrm{w}}, \tau_{0}^{+}=\tau_{\mathrm{w}} / \tau_{\mathrm{w} 0}$, where $\tau_{\mathrm{w} 0}$ is the wall shear stress in case where the non-adiabatic effects are not considered, and $\mathrm{B}=2 \vartheta_{0} /\left(\mathrm{c}_{\mathrm{f}} \mathrm{u}_{\infty}\right)$ is the so called "blowing parameter". Additionally, $\vartheta_{0}$ denotes the transverse velocity, which in case of condensation or boiling is equal to $\mathrm{q}_{\mathrm{w}} /\left(\mathrm{h}_{\mathrm{lv}} \rho_{\mathrm{l}}\right)$. In case of small values of $\mathrm{B}$ the relation (4) reduces to the form:

$$
\tau_{0}^{+}=\left(1 \pm \frac{B}{2}\right)
$$

The blowing parameter is hence defined as:

$$
B=\frac{2 v_{0}}{c_{f} u_{\infty}}=\frac{2 q}{c_{f 0}\left(u_{G}-u_{L}\right) h_{l v} \rho_{G}}=\frac{2 q \frac{\rho_{L}}{\rho_{G}}}{c_{f 0} G(s-1) h_{l v}}
$$

\subsection{Model of blowing parameter}

Analysis of the liquid and vapor phase is based on examination of mass and momentum balance equations with respect to the non-adiabatic effect influence. Fig. 1 shows the considered schematic of the annular flow model. The analysis will be conducted with the reference to condensation. Conservation of mass requires that the 
mass flow rate of liquid in the film, liquid in the form of droplets in the core and vapor in the core is constant:

$$
B=\frac{2 v_{0}}{c_{f} u_{\infty}}=\frac{2 q}{c_{f 0}\left(u_{G}-u_{L}\right) h_{l v} \rho_{G}}=\frac{2 q \frac{\rho_{L}}{\rho_{G}}}{c_{f 0} G(s-1) h_{l v}}
$$

In the model presented below the following notation is used. The liquid film cross-section area on the wall is expressed by the expression $\mathrm{A}_{\mathrm{f}}=\pi \mathrm{D} \delta_{\mathrm{f}}$, while the core cross-section area as $A_{c}=\pi\left(D-\delta_{f}\right)^{2} / 4$. The wetted perimeter is given by the relation $\mathrm{P}_{\mathrm{f}}=\pi \mathrm{D}$, where $\mathrm{D}$ is the channel inner diameter. The mean liquid film velocity is given as $\mathrm{U}_{\mathrm{f}}=\dot{m} /\left(\rho_{\mathrm{f}} \mathrm{A}_{\mathrm{f}}\right)$. Authors assumed that the interfacial velocity can be determined from the relationship $U_{i}=2 U_{f}$.
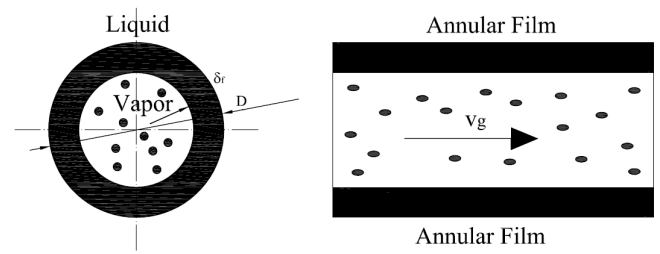

Figure 1. 1Annular flow structure model.

\section{Mass balance in liquid film and core} Liquid film:

$$
\frac{\mathrm{dm}_{\mathrm{f}}}{\mathrm{dz}}=-\Gamma_{\mathrm{lv}}+\mathrm{D}-\mathrm{E}
$$

Two-phase flow vapor core:

$$
\frac{d m_{c d}}{d z}=-D+E
$$

Vapor in vapor core:

$$
\frac{d m_{c v}}{d z}=-\Gamma_{l v}
$$

In (8) and (9) the terns $\mathrm{D}$ and $\mathrm{E}$ denote deposition and entrainment in the annular flow. The remaining term in equation, $\Gamma_{\mathrm{lv}}=\mathrm{q}_{\mathrm{w}} \mathrm{P} / \mathrm{h}_{\mathrm{lv}}$, is responsible for the condensation of vapor. Concentration of droplets in the core is defined as a ratio of mass flow rate droplets in the core to the sum of mass flow rate vapor and entrained liquid droplets from the flow.

$$
C=\frac{\dot{m}_{c f}}{\dot{m}_{c v} v_{g}+\dot{m}_{e f} v_{f}}
$$

The combined mass flow rate of the core results from combination of (9) and (10):

$$
\frac{d m_{c}}{d z}=-\Gamma_{l v}-D+E
$$

The amount of entrained droplets in (11) can be determined from the mass balance:

$$
\dot{m}_{e f}=\dot{m}-\dot{m}_{f}-\dot{m}_{c v}
$$

\section{Momentum balance in liquid film and core}

The change of momentum is mainly due to the mass exchange between the core of flow and liquid film (evaporation, droplet deposition or entrainment). Acceleration is neglected. The flow schematic is shown in Figure 2.

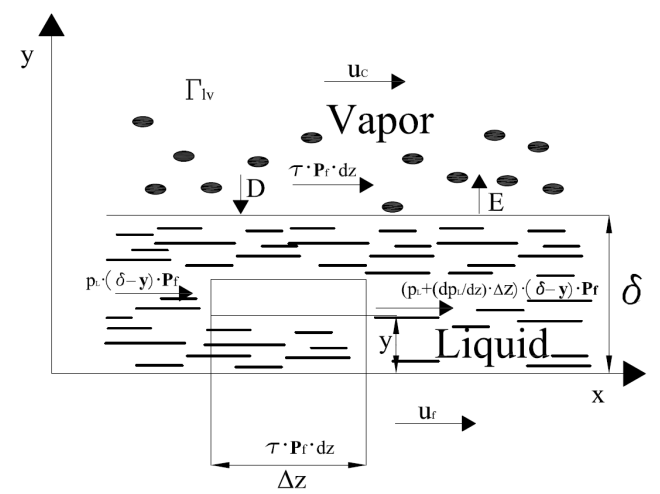

Figure 2Flow schematic for the momentum in the liquid film.

\section{Momentum equation for liquid film}

Momentum equation for the liquid film reads:

$$
\begin{aligned}
& -\frac{d p_{L}}{d z} \Delta z \cdot(\delta-y) \cdot P_{f}-\tau P_{f} \Delta z+\tau_{i} P_{f} \Delta z= \\
& \left(\Gamma_{l v} u_{i}+D u_{c}-E u_{i}\right) \cdot \Delta z
\end{aligned}
$$

Pressure gradient in the liquid film is therefore (assuming that $\rho_{\mathrm{f}}=\rho_{\mathrm{l}}$ and $\left.\mu_{\mathrm{f}}=\mu_{\mathrm{l}}\right)$ :

$$
-\left(\frac{d p_{l}}{d z}\right)=\frac{3 \mu_{f} \dot{m}_{f}}{P_{f} \rho_{f} \delta^{3}}-\frac{3 \tau_{i}}{2 \delta}+\frac{3\left(\Gamma_{l v} u_{i}+D u_{f}-E u_{i}\right)}{2 \delta P_{f}}
$$

\section{The momentum balance for the core flow}

Control volume for the flow core is shown in Fig. 3 where momentum equation for the mixture in the core is given by equation:

$$
\begin{aligned}
& \rho_{T P} u_{c}^{2} A_{c}+\frac{d}{d z}\left(\rho_{T P} u_{c}^{2} A_{c}\right) \Delta z-\rho_{T P} u_{c}^{2} A_{c}+ \\
& {\left[-\Gamma_{l v} u_{i}-D u_{c}+E u_{i}\right] \Delta z} \\
& =p_{v} A_{c}-\left[p_{v} A_{c}+\frac{d\left(p_{v} A_{c}\right)}{d z} \Delta z\right]-\tau_{i} P \Delta z
\end{aligned}
$$

Interfacial shear stress are: 


$$
\begin{gathered}
\tau_{i}=\frac{1}{P}\left[A_{c}\left(-\frac{d p_{v}}{d z}\right)-p_{v} \frac{d A_{c}}{d z}\right]-\frac{1}{P} \frac{d}{d z} \\
\left(\rho_{T P} u_{c}^{2} A_{c}\right)-\frac{1}{P}\left(-\Gamma_{l v} u_{i}-D u_{c}+E u_{i}\right)
\end{gathered}
$$

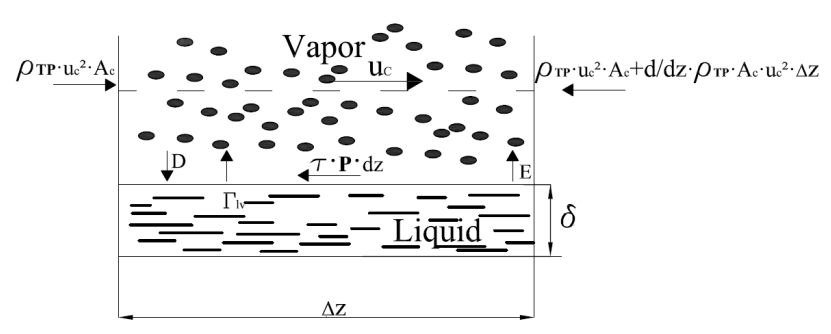

Figure 3 Control volume for the flow core

The relationship expresses the interfacial shear stress for the two-phase flow (here condensation), and included are the non-adiabatic effects: liquid film evaporation, droplets deposition and entrainment. For the case of no evaporation of the liquid film, but presence of entrainment and deposition, the interfacial shear stress distribution takes the form :

$$
\begin{gathered}
\tau_{i o}=\frac{-\frac{1}{A_{c}}\left(-D u_{c}+E u_{i}\right)+\frac{3 \mu_{f} \dot{m}_{f}}{P_{f} \rho_{f} \delta^{3}}}{\frac{P_{f}}{A_{c}}+\frac{3}{2 \delta}} \\
+\frac{\frac{3}{2 \delta P_{f}}\left(-D u_{f}+E u_{i}\right)}{\frac{P_{f}}{A_{c}}+\frac{3}{2 \delta}}
\end{gathered}
$$

In this case we can neglect the entrainment and deposition i.e. by assigning $\mathrm{E}=0$ and $\mathrm{D}=0$, we obtain a simplified form of the diabatic two-phase flow effect in the form:

$$
\frac{\tau_{i}}{\tau_{i o}}=1+\frac{2 q_{w} \delta\left(\frac{4 \delta}{D}+\frac{3}{2}\right)}{3 \mu_{f} h_{l v}}=(1+B)
$$

\section{Non-adiabatic effects in other flows}

In case of the non-adiabatic effects in other than annular structures the two-phase flow multiplier, which incorporates the non-adiabatic effect, can be presented as follows, Mikielewicz (2010):

$$
\Phi_{T P B}^{2}=\frac{\xi_{T P B}}{\xi_{0}}=\sqrt{\Phi^{2}+\frac{\xi_{P B}^{2}}{\xi_{0}^{2}}}=\Phi^{2} \sqrt{1+\frac{\left(\frac{8 \alpha_{P B} d}{\lambda \operatorname{Re} P r}\right)^{2}}{\xi_{0}^{2} \Phi^{2}}}
$$

The two-phase flow multiplier presented in equation (20) reduces to adiabatic formulation in case when the applied wall heat flux is tending to zero.
Generalizing the obtained above results it can be said that the two-phase flow multiplier inclusive of nonadiabatic effects can be calculated, depending upon the particular flow case and the flow structure in the following way:

$$
\begin{aligned}
& \Phi^{2}{ }_{T P C}=\Phi^{2}{ }_{T P B}=\frac{\xi_{T P B}}{\xi_{0}}= \\
& \left\{\begin{array}{l}
\Phi^{2}\left(1 \pm \frac{B}{2}\right) \text { for annular structure, condensati on } \\
\text { and boiling } \\
\Phi^{2} \sqrt{1+\left(\frac{8 \alpha_{P B} D}{\lambda \operatorname{Re} \operatorname{Pr} \xi_{0} \Phi^{2}}\right)^{2}} \text { for other flow structures }
\end{array}\right.
\end{aligned}
$$

In (21) there is no specification of which two-phase flow multiplier model should be applied. That issue is dependent upon the typ e of considered fluid. Author's up to date experience shows that the influence of the twophase flow multiplier is very important and each fluid could have a different description of a two-phase resistance, D. Mikielewicz [6]. In the form applicable to conventional and small diameter channels the MullerSteinhagen and Heck model yields, [6]:

$$
\Phi^{2}=\left[1+2\left(\frac{1}{f_{1}}-1\right) x \operatorname{Con}^{m}\right] \cdot(1-x)^{1 / 3}+x^{3} \frac{1}{f_{1 z}}
$$

where $\operatorname{Con}=\left(\sigma / g /\left(\rho_{\mathrm{L}}-\rho_{\mathrm{G}}\right)\right)^{0.5} / \mathrm{d}$ and $\mathrm{m}=0$ for conventional channels. Best consistency with experimental data, in case of small diameter and minichannels, is obtained for $m=-1$. In $(22) f_{1}=\left(\rho_{L} / \rho_{G}\right)\left(\mu_{L} / \mu_{G}\right)^{0.25}$ for turbulent flow and $\mathrm{f}_{1}=\left(\rho_{\mathrm{L}} / \rho_{\mathrm{G}}\right)\left(\mu_{\mathrm{L}} / \mu_{\mathrm{G}}\right)$ for laminar flows. Introduction of the function $\mathrm{f}_{1 \mathrm{z}}$, expressing the ratio of heat transfer coefficient for liquid only flow to the heat transfer coefficient for gas only flow, is to meet the limiting conditions, i.e. for $\mathrm{x}=0$ the correlation should reduce to a value of heat transfer coefficient for liquid, $\alpha_{\mathrm{TPK}}=\alpha_{\mathrm{L}}$ whereas for $\mathrm{x}=1$, approximately that for vapor, i.e. $\alpha_{\mathrm{TPK}} \cong \alpha_{\mathrm{G}}$. Hence:

$$
f_{1 z}=\frac{\alpha_{G O}}{\alpha_{L O}}
$$

where $f_{1 z}=\left(\lambda_{G} / \lambda_{L}\right)$ for laminar flows and for turbulent flows $\mathrm{f}_{1 \mathrm{z}}=\left(\mu_{\mathrm{G}} / \mu_{\mathrm{L}}\right)\left(\lambda_{\mathrm{L}} / \lambda_{\mathrm{G}}\right)^{1.5}\left(\mathrm{c}_{\mathrm{pL}} / \mathrm{c}_{\mathrm{pG}}\right)$.

\section{Comparision with experimental data}

On of the objectives of this study is to add data of HFE 7100 and HFE 7000 for mini channels because of the lack in published studies. This data is greatly interesting because of the very different thermo physical properties of such fluids compared to other substances commonly tested in mini channels. To include this data will therefore be valuable to receipt propriety author's general model for pressure drop in boiling and condensation in 
mini and micro channels. Another reason for understanding the behavior of two phase flow of the working fluids HFE 7100 and HFE 7000 is due to increased concerns of ozone depletion(ODP) and GWP (global warming potential), as increased knowledge of the performance of this fluids may contribiute to HCFC and HFC refrigerants and may use in many other perspective ecological application such as organic Rankine cycles. A $2.23 \mathrm{~mm}$ circular minichannel is used to measured both two-phase pressure losses of the fluids HFE7100 and HFE7000. The pressure drops tests have been performed in diabatic flow conditions. An experimental rig for the examination of condensation in flow was realized as a development and modification of the previous installation designed for the research on flow boiling and boiling crises. The great advantage of the modernized experimental facility is the possibility of processing both flow boiling and flow condensation researches simultaneously.

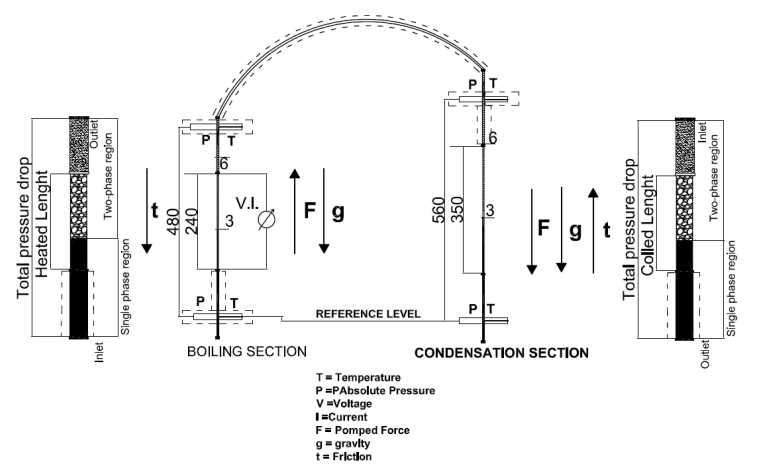

Figure 4 Diagram of experimental facility

The rig is intended to work with any low boiling point fluid with the estamated heat of vaporization not exceeding $200 \mathrm{~kJ} / \mathrm{kg}$. It's flow is forced by an electrically-powered pump, which is capable to provide the mass flowrate from 0,5 to $10 \mathrm{~g} / \mathrm{s}$ and the overpressure up to 3 bars. The gear pump has been chosen to provide circulation of fluid in the test section and to avoid any flow pulsations. Adjustment of the mass flow rate is realized by changing voltage of the pump's power supply or using by-pass. Test fluid is pumped from the main tank through the Danfoss mass flowmeter type MASS D1 3 working with MASS 6000 19" IP20 interface. This system, called the MASSFLO, returns $0.3 \%$ accuracy of the full scale. Subsequently the medium goes to the evaporation section. Heating of the test fluid is realized in the vertical silver tube of $2.3 \mathrm{~mm}$ inner diameter, identical as in the condensation section, powered by low voltage, high current DC power supply. It gives up to 1.2 $\mathrm{kW}$ of heating power and up to $168 \mathrm{~kW} / \mathrm{m}^{2}$ of the heat flux. In this way, the full range of quality is possible to be obtained. Current, voltage, inlet and outlet temperatures and pressures are measured to determine the heat flux and quality. The distribution of tube wall temperature can be registered using the infrared camera. That means that the full range research on flow boiling and boiling crises can also be carried out on the discussed facility. After obtaining the appropriate parameters, the fluid goes from the pre-heater directly to the condensation section. In this experiment a silver tube of $2.3 \mathrm{~mm}$ inner diameter and the length of $350 \mathrm{~mm}$ has been used. The test fluid flows into the tube with pre-defined $\mathrm{x}$ and is cooled by cold water flowing around an external tube wall to get the expected cooling conditions. Cooling is accomplished by a unique system of many separated parallel channels instead of a typical pipe-in-pipe configuration. This method ensures constatnt heat flux taken from the tube wall and allows to measure a local increase of a cooling medium temperature. Leaving single channels out of the flow allows for the full separation of thermocouples from the cooling medium. The mass flux, inlet and outlet temperatures of the cooling water are measured to determine the heat flux. Distribution of the tube wall temperature is registered using the set of 10 T-type thermocouples. The thermocouples placed in each water channel allow to determine the local increase of a cooling water temperature. From the condensation section the test fluid goes back to the main tank. The unit is also equipped with an additional heat exchanger in the main tank. It serves to additional heating or cooling of the medium depending on the needs.

Examples of comparision own model with in-house experimental data for HFE7010 are presented in Fig. 5-7 and for HFE 7000 in Fig 8-9.

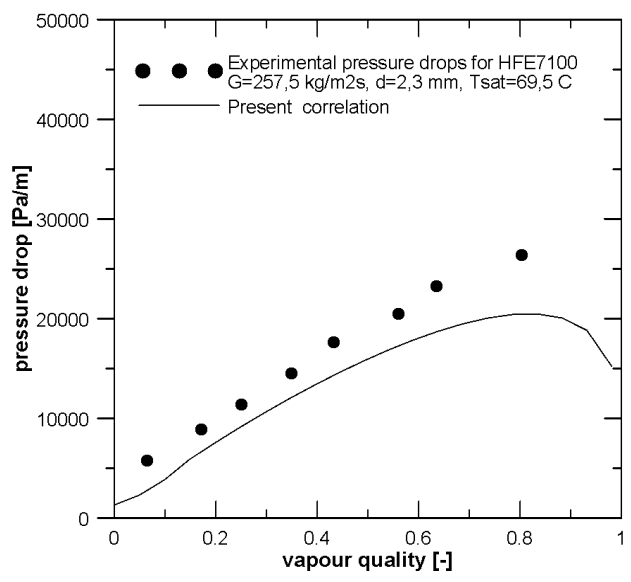

Figure 5 Pressure drop distribution in function of quality, HFE $7100, \mathrm{G}=257,5 \mathrm{~kg} / \mathrm{m}^{2} \mathrm{~s}, \mathrm{~d}=2.3 \mathrm{~mm}$, Tsat $=69,5^{\circ} \mathrm{C}$, own data

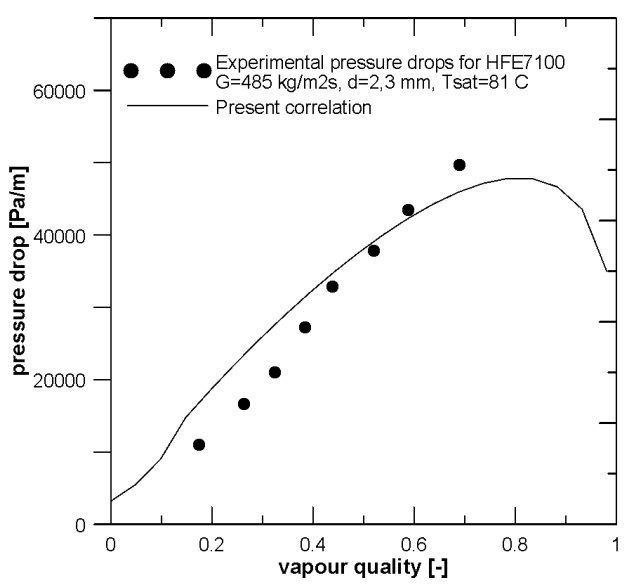

Figure 6 Pressure drop distribution in function of quality, HFE $7100, \mathrm{G}=485 \mathrm{~kg} / \mathrm{m}^{2} \mathrm{~s}, \mathrm{~d}=2.3 \mathrm{~mm}$, Tsat $=81^{\circ} \mathrm{C}$, own data 


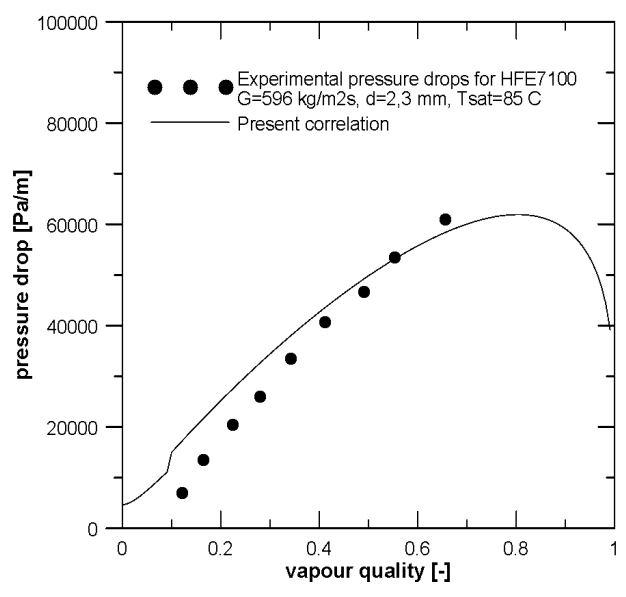

Figure 7 Pressure drop distribution in function of quality, HFE $7100, \mathrm{G}=485 \mathrm{~kg} / \mathrm{m}^{2} \mathrm{~s}, \mathrm{~d}=2.3 \mathrm{~mm}$, Tsat $=81^{\circ} \mathrm{C}$, own data

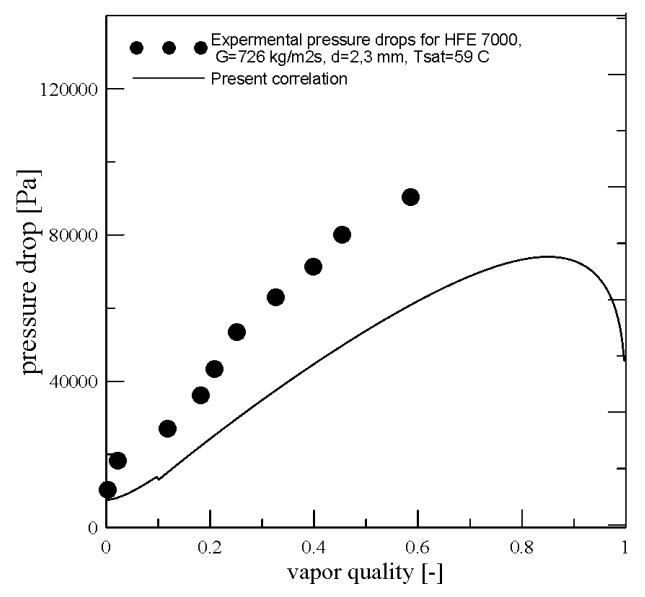

Figure 8 Pressure drop distribution in function of quality, HFE $7000, \mathrm{G}=726 \mathrm{~kg} / \mathrm{m} 2 \mathrm{~s}, \mathrm{~d}=2.3 \mathrm{~mm}$, Tsat $=59^{\circ} \mathrm{C}$, own data

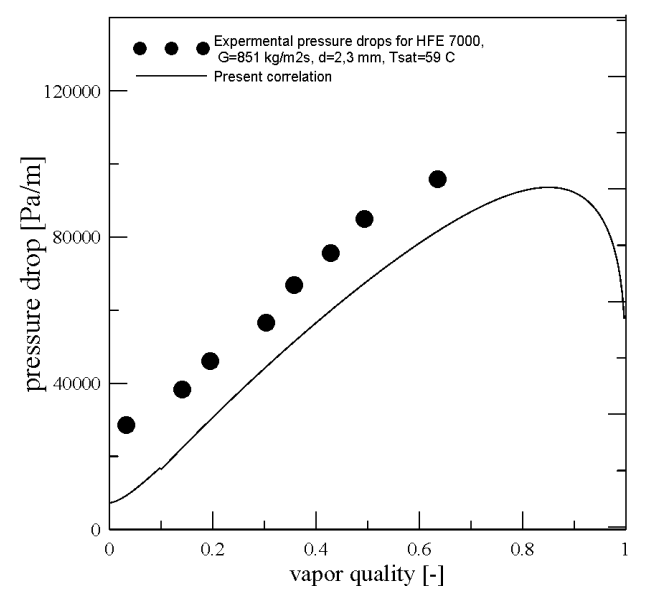

Figure 9 Pressure drop distribution in function of quality, HFE $7000, \mathrm{G}=851 \mathrm{~kg} / \mathrm{m} 2 \mathrm{~s}, \mathrm{~d}=2.3 \mathrm{~mm}$, Tsat $=59^{\circ} \mathrm{C}$, own data

\section{Conclusions}

In the paper some issues of frictional term of pressure drop modeling in minichannels with change of phase were presented. Results were compared with own experimental data for new generation fluids: HFE 7100 and HFE 7000. The model of flow boiling and flow condensation presented in the paper is a general model, which can be advised for use in engineering applications. The model is general as it enables to be included into any two-phase flow multiplier definition. Satisfactory consistency of discussed model with own experimental data for condensation has been found. In author's opinion, the presented model can be suggested for a wider use amongst engineers, but further validation with experimental data would add value to its robustness.

\section{References}

1. T.Bohdal, H.Charun, M.Sikora, Comparative investigations of the condensation of R134a and $\mathrm{R} 404 \mathrm{~A}$ refrigerants in pipe minichannels, International Journal of Heat and Mass Transfer, 54, 1963-1974, (2011)

2. D.Mikielewicz, J.Mikielewicz, A common method for calculation of flow boiling and flow condensation heat transfer coefficients in minichannels with account of nonadiabatic effects, Heat Tr. Engng, 32, 1173-1181, (2011).

3. A.Cavallini, , G. Censi, D. Del Col, L. Doretti, G.A. Longo, Rossetto L., Condensation of Halogenated Refrigerants inside Smooth Tubes, HVAC and Research, vol. 8, 429-451, 2002.

4. J.R. Thome, J. El Hajal, A. Cavallini,Condensation in horizontal tubes, part 1: two-phase flow pattern map, International Journal of Heat and Mass Transfer, vol.46, pp.3349-3363, (2003)

5. J. Mikielewicz: Semi-empirical method of determining the heat transfer coefficient for subcooled saturated boiling in a chan., Int. J. Heat Transfer, 17, 1973, 1129-1134.

6. D.Mikielewicz, A new method for determination of flow boiling heat transfer coefficient in conventional diameter channels and minichannels, Heat Transfer Eng., Vol. 31 (4), 276-284, 2009. 